\title{
Projeto robusto de parâmetros em sistemas sinal-resposta: comparação de métodos de modelagem e análise
}

\author{
Marta Afonso Freitas \\ Rosiane Mary Rezende Faleiro \\ Marco Fábio Borges \\ UFMG
}

\begin{abstract}
Resumo
A maior parte da literatura relacionada a Projeto Robusto de Parâmetros (RPD), uma metodologia introduzida por Taguchi (1986), envolve situações nas quais a característica de qualidade de interesse (resposta) é uma quantidade única que possui um valor ótimo especificado. Uma tendência recente em aplicações industriais de RPD consiste em considerar sistemas complexos, os quais são chamados de "sistemas com características dinâmicas" ou apenas "sistemas dinâmicos", na terminologia de Taguchi, ou por "sistemas sinal-resposta", neste artigo. Recentemente, métodos de modelagem e análise para o problema de RPD para sistemas sinal-resposta têm sido desenvolvidos. Neste artigo, três abordagens de modelagem são comparadas através de um exemplo real: Modelagem da Medida de Desempenho (PMM), Modelagem da Resposta (RM) e Modelagem da Função Resposta (RFM). Mostra-se que a abordagem RFM permite uma maior flexibilidade para investigar efeitos de fatores para o experimento RPD para sistemas sinal-resposta.
\end{abstract}

Palavras-chave

Projeto robusto de parâmetros, sistemas sinal-resposta, razão sinal-ruído.

\section{Robust parameter design in signal-response systems: comparison of modeling and analysis methods}

\begin{abstract}
Most of the literature concerning robust parameter design (RPD], a methodology introduced by Taguchi (1986), involves situations where the quality characteristic of interest (response) is a single quantity which has a specified optimal value. A recent trend in the industrial applications of RPD consists in consider complex systems which are called "systems with dynamic characteristics" or "dynamic systems", in Taguchi's terminology, or "signal-response systems", in this paper. Recently, modeling and analysis methods for the dynamic system have been developed. In this paper, three modeling approaches are compared by use of a real example: Performance Measure Modeling (PMM], Response Modeling (RM) and Response Function Modeling [RFM]. It is shown that the RFM approach allows greater flexibility to investigate factor effects for the RPD experiment.
\end{abstract}

Key words

Robust parameter design, signal-response systems, signal-noise ratio. 


\section{INTRODUCÃ̃O}

\section{Conceito de Projeto Robusto de Parâmetros}

O Projeto Robusto de Parâmetros (Robust Parameter Design - RPD), é na verdade um conceito de Engenharia, cuja importância foi trazida à tona e à discussão por Taguchi (1986) e motivou o desenvolvimento de uma metodologia com o mesmo nome. O que se busca com o RPD é desenvolver um projeto (do ponto de vista da Engenharia) de produto ou processo tal que os parâmetros especificados no mesmo o torne robusto aos efeitos dos fatores que causam a variabilidade no desempenho. A literatura está repleta de trabalhos apresentando a metodologia, discutindo e propondo procedimentos alternativos. Destacam-se Nair (1992) que apresenta um painel de discussão em torno do tema, reunindo opiniões de vários pesquisadores de renome internacional: Box (1988), Phadke (1989), Kacker (1985), Nair e Shoemaker (1990) e Wu e Hamada (2000).

apropriados dos fatores $\mathrm{X}$, nos quais o desempenho do sistema é robusto (menos afetado) pela variação incontrolável dos fatores de ruído N. Esta é, sem dúvida, a maior contribuição de Taguchi nesta área, pois a divisão entre fatores de controle e ruído já era feita anteriormente na abordagem estatística para planejamento de experimentos, e o que muda aqui é a forma de se olhar para os fatores de ruído, tentandose agora incorporá-los na experimentação.

\section{Aplicações da Metodologia RPD: sistemas de resposta única e sistemas sinal-resposta}

A maior parte da literatura estatística referente à metodologia de RPD envolve situações onde a característica de qualidade de interesse (resposta) é uma quantidade única $\mathrm{Y}$, que tem um valor ótimo especificado. Como exemplo, Shoemaker, Tsui e Wu (1991) apresentaram um estudo de caso envolvendo a formação de camadas de silício no recobrimento de wafers utilizados na confecção de placas de circuito impresso. Neste caso, a resposta Y era a espessura da camada de recobrimento, cujo valor alvo especificado foi $14,5 \mu \mathrm{m}$. Taguchi denominou este tipo de aplicação como sendo o de um sistema com característica de qualidade estática ou simplesmente sistema estático, enquanto Miller e Wu (1996) utilizaram o termo sistema de resposta única. Historicamente, a

Na abordagem de Taguchi para o RPD, um processo ou produto é considerado um sistema que visa a transformação de energia para cumprir uma determinada função. As entradas (inputs) do sistema são divididas em: (a) parâmetros ou fatores de controle " $\mathrm{X}$ " - parâmetros que podem ser facilmente controlados e manipulados durante a produção ou operação - e (b) fatores ou variáveis de ruído "N" - variáveis que influenciam na funcionalidade do sistema cujo monitoramento e controle durante a produção ou operação é difícil e/ou muito oneroso. Dentro desta perspectiva, assume-se que em todo sistema de engenharia existem formas de relação perfeita ou ideal entre a entrada e a saída do sistema, na qual toda energia aplicada (de entrada) é transformada eficientemente em energia para cumprir a função desejada para o sistema. Quando parte dessa energia é perdida (ou seja, a eficiência do sistema é menor que $100 \%$ ), transforma-se em alguma outra forma de energia indesejável, responsável pelos sintomas ou problemas que se busca minimizar: ruídos, vibrações, trincas, desgastes, etc. Portanto, quando se melhora um sistema, aumentando a relação entre a energia útil gerada e a energia que o alimenta, haverá menos "perda" e, portanto, menor incidência de sintomas indesejáveis.

Assim, a idéia básica do RPD é identificar os set-ups literatura tem dado maior ênfase a este tipo de problema. Nair (1992) apresenta uma lista exaustiva de referências relacionadas a este tema. Outras referências importantes e mais recentes não incluídas em Nair (1992) são Lee e Nelder (2003) que apresentam uma modelagem via modelos lineares generalizados, McCaskey e Tsui (1997), Engel e Huele (1996) e Greco (1993).

O segundo tipo de aplicação da metodologia RPD referese a situações onde a resposta $\mathrm{Y}$ assume diferentes valores como resultado de mudanças de um fator sinal M. É denominado por Taguchi como sistemas com características dinâmicas ou apenas, sistemas dinâmicos. Miller e Wu (1996) consideraram que o termo dinâmico poderia gerar dúvidas, pois há a mesma terminologia para modelos físicos e matemáticos. Por esta razão adotaram a terminologia sistema sinal-resposta para este tipo de situação, porque é a relação entre sinal e resposta que é de importância. Este é o termo adotado neste artigo. O desempenho de um sistema deste tipo só pode ser avaliado levando-se em conta a relação entre a resposta Y e o sinal M. DeMates (1990) descreve um experimento relacionado ao sistema de moldagem de partes por injeção, o qual tinha como função injetar quantidades diferentes de material dependendo do tipo de parte a ser moldada e a sua aplicação. Portanto, era necessário obter 
um método confiável para controlar a quantidade de material injetado. O peso da parte a ser produzida foi adotado como resposta $(\mathrm{Y})$ e a pressão alta de injeção foi escolhida como o sinal M, devido a sua capacidade já conhecida de modificar a quantidade de material injetada e, conseqüentemente, o peso da parte moldada. $\mathrm{O}$ experimento foi realizado com sete fatores de controle, dois fatores de ruído (do tipo combinado) e vários valores de $\mathrm{M}$. O objetivo era encontrar o melhor set-up dos fatores de controle tal que a relação entre $\mathrm{Y}$ e $\mathrm{M}$ fosse o menos afetada possível pelo efeito dos fatores de ruído. Desta forma, mesmo com o efeito dos fatores de ruído, o peso da parte obtida através do sistema poderia ser controlado no dia-a-dia da produção de maneira eficiente.

Neste artigo, considera-se com detalhe o experimento descrito por Borges e Freitas (2005), no qual o objetivo era modificar o projeto de um cabo comando de embreagem. $\mathrm{O}$ problema identificado no sistema foi o surgimento de um ruído baixo perceptível ao motorista, acompanhado de vibração durante o desacoplamento da embreagem, através da liberação do pedal de embreagem, após as trocas de marchas. Esta situação prática se enquadra no tipo sinal-resposta, conforme será visto posteriormente, e está descrita na seção a seguir.

$\mathrm{O}$ interesse em problemas relacionados a sistemas sinalresposta vem crescendo, uma vez que várias situações encontradas na prática estão dentro desta categoria. Recentemente, a literatura tem apresentado vários trabalhos relacionados à modelagem e análise de sistemas do tipo sinal-resposta, como por exemplo Bae e Tsui (2006), Nair, Taam e Ye (2002), Miller (2002), Wilkins (2000), Taguchi, Chowdhury e Taguchi (2000), Wu e Hamada (2000, capítulo 11), Tsui (1999), Fowkes \& Creveling (1995, p. 97), Grove e Davis (1992, p. 329), Phadke (1987, p. 214) e Ross (1988).

É importante destacar que, para um sistema sinal-resposta, o conceito de qualidade ideal é baseado na relação (função) ideal entre o sinal e a resposta. A perda de qualidade, neste caso, é causada por desvios da relação ideal. $\mathrm{Na}$ situação abordada neste artigo, a relação ideal entre o sinal e a resposta é do tipo linear simples. Um ganho significante de qualidade pode ser atingido definindo-se primeiramente a função ideal do sistema e posteriormente utilizando experimentos planejados para buscar o set-up ótimo - o que minimiza os desvios. Entretanto, a solução "ótima" obtida pode ser diferente, dependendo da abordagem utilizada para modelagem e análise de sistemas deste tipo. Três propostas de modelagem foram encontradas na literatura: (1) Modelagem da Medida de Desempenho (Performance Measure Modeling - PMM), também conhecida por Modelo de Perda (Loss Model); (2) Modelagem da Resposta (Response Modeling - RM); e (3) Modelagem da Função Resposta (Response Function Modeling - RFM). Este artigo compara as três abordagens de análise para sistemas sinal-resposta utilizando os dados do experimento para o cabo comando de embreagem apresentado na próxima seção.

A seguir, é apresentada a classificação dos sistemas sinal-resposta em dois tipos: sistemas de medição e sistemas com múltiplos alvos, a qual é importante para a escolha da medida de desempenho (MD) adequada para otimizar um dado sistema sinal-resposta. Na seção seguinte, são apresentadas algumas medidas de desempenho (MDs) sugeridas na literatura para cada tipo de sistema sinal-resposta, e são apresentadas as três abordagens de análise. Finalmente, os dados do experimento para o cabo comando de embreagem são reanalisados, utilizando as três abordagens descritas anteriormente, e são feitas as considerações finais.

\section{EXPERIMENTO PARA ELIMINAC̣ÃO DO RUÍDO NO NOVO CABO COMANDO DE EMBREAGEM}

Borges e Freitas (2005) apresentaram um estudo envolvendo o desenvolvimento de um novo cabo de comando de embreagem para substituir o cabo que já estava em utilização em um veículo em comercialização e apresentava um histórico de reclamações dos clientes junto à rede assistencial, devido a um problema de ruptura.

$\mathrm{Na}$ etapa final de desenvolvimento, este novo cabo foi submetido a um teste subjetivo denominado Quality Profile, realizado conforme uma escala SAE (Society of Automotive Engineers) de 1 a 10, sendo a nota mínima de aprovação 6 e, quanto maior a nota, melhor o desempenho do produto quanto ao quesito avaliado. Neste teste, um piloto de testes da empresa examina o comportamento do veículo com o novo cabo em vias públicas (trânsito urbano e rodovia), com ótica do cliente final. Entretanto, enquanto realizava o teste, o piloto identificou um problema que era sinalizado através de um ruído baixo acompanhado de vibração durante o desacoplamento da embreagem, através da liberação do pedal de embreagem, após as trocas de marchas. Percebeu-se que este ruído era devido à transferência de vibração do motor para a posição do motorista no interior do veículo através do cabo de embreagem. Para validação do teste e efeito comparativo, foram testados, além do veículo com o novo cabo, mais três veículos normais de produção. Para o problema mencionado, o veículo com o novo cabo recebeu nota 4 enquanto que os demais veículos normais de produção receberam nota 6 . Portanto, o novo cabo de embreagem proposto apresentava um nível de qualidade, neste requisito, menor que aquele que estava por substituir.

Após o controle detalhado de todas as características de projeto do produto, constatou-se que as especificações do novo cabo estavam totalmente dentro das especificações de engenharia e com um rendimento de $82 \%$, valor este dentro do especificado (o mínimo aceitável era de $80 \%$ ). Portanto, devido ao fato de apresentar um nível de desempenho 
menor do que aquele que seria substituído, sua adoção foi interrompida até a resolução do problema. Decidiu-se, então, planejar um experimento do tipo RPD com o objetivo de eliminar a vibração e o ruído transmitidos para dentro do veículo pelo novo cabo de embreagem, após as mudanças de marcha, e obter uma nota SAE igual ou superior a 6 no teste Quality Profile.

\section{Descrição do Sistema}

A função básica do sistema em estudo é transmitir a força realizada sobre o pedal pelo motorista para a alavanca da embreagem, com a finalidade de desacoplar o platô do disco de embreagem, possibilitando as trocas de marchas.

A variável resposta $(\mathrm{Y})$ foi definida como a força de acionamento aplicada na alavanca da embreagem (Kgf) força que chega ao final do cabo para acionamento da embreagem; energia que sai do sistema - e o sinal (M) foi definido como a força aplicada pelo motorista no pedal de embreagem (Kgf) - energia que entra no sistema. A relação ideal para o sistema é linear, com intercepto 0 e inclinação 1, na qual toda força aplicada pelo motorista no pedal de embreagem para o desacoplamento da embreagem deveria ser transmitida para a alavanca de acionamento da embrea- gem, sem perdas. Quatro níveis foram selecionados para o fator sinal: 15, 30, 45 e 60 (Kgf). Portanto, seguindo a classificação apresentada, este sistema é do tipo sinal-resposta com múltiplos alvos (veja a seção "Classificação dos sistemas sinal-resposta"). Através de reuniões realizadas com o fornecedor, foram identificados os fatores de controle relevantes e seus respectivos níveis, os quais podem ser vistos na Tabela 1, e uma condição ruído que representa a combinação de dois fatores de ruído, que pode ser vista na Tabela 2. Os fatores de ruído foram estudados em conjunto, e não de forma separada, a fim de reduzir o número total de protótipos a ser construídos para o experimento.

$\mathrm{O}$ experimento foi planejado segundo um arranjo ortogonal L8, que possibilita a exploração de sete fatores de controle a dois níveis cada um. Os protótipos foram construídos em um dia na linha produtiva do fornecedor. Foram produzidos dois protótipos de cada configuração da Tabela 3: um na condição ruído $\mathrm{N}=+1$ e outro na condição $\mathrm{N}=-1$, totalizando 16 protótipos.

Os sintomas que podem ser gerados devido à perda de energia do sistema são exatamente os problemas que podem ser encontrados e acarretar insatisfação no cliente final: ruído, vibração (aspereza), desgaste do tubo e do cabo, ruptura do cabo e força no pedal de embreagem.

Tabela 1: Fatores de controle do experimento do novo cabo de embreagem.

\begin{tabular}{|c|c|c|}
\hline \multirow{2}{*}{ FATOR DE CONTROLE } & \multicolumn{2}{|c|}{ NÍVEIS } \\
\hline & $(+1)$ & $(-1)$ \\
\hline A - Fornecedor da Boccola & A1 & $\mathrm{A} 2$ \\
\hline B - Fornecedor do Tasselo & B1 & $\mathrm{B} 2$ \\
\hline C - Terminal & Com ângulo & Reto \\
\hline D - Layout & Atual & Modificado \\
\hline E - Tubetto & Não Passante & Passante \\
\hline F - Material do Tubetto & Poliacetal & Polietileno \\
\hline G - Massa Smorzante & Sem & Com \\
\hline
\end{tabular}

Obs: (+1) indica o nível alto do fator; (-1) nível baixo.

Tabela 2: Níveis da condição ruído do experimento do novo cabo de embreagem.

\begin{tabular}{|c|c|c|}
\hline $\begin{array}{c}\text { QUANTIDADE DE GRAXA NO } \\
\text { TUBETTO }\end{array}$ & TEMPERATURA & $\begin{array}{c}\text { NíVEL DO FATOR DE RUíDO } \\
\text { COMBINADO (N) }\end{array}$ \\
\hline 2 gramas & 30 graus & +1 \\
\hline Sem graxa & 60 graus & -1 \\
\hline
\end{tabular}




\section{Coleta de dados}

Todos os 16 protótipos foram testados, tiveram a curva de rendimento levantada e a força no final do cabo foi extraída para as forças de 15:30: 45 e 60, conforme definido anteriormente. Os dados, registrados na Tabela 4 , foram processados e analisados por Borges e Freitas (2005) utilizando a razão sinal-ruído dinâmica de Taguchi e serão reanalisados na seção "Modelagem e análise de sistemas sinal-resposta: as abordagens PMM, RM e RFM" utilizando as três metodologias mencionadas: PMM, RM e RFM.

Tabela 3: Configurações dos protótipos no experimento do novo cabo de embreagem.

\begin{tabular}{|c|c|c|c|c|c|c|c|}
\hline CONFIGURAÇÃO & BOCGOLA & TASSELO & TERMINAL & LAYOUT & TUBETTO & $\begin{array}{c}\text { MATERIAL } \\
\text { DUBETTO }\end{array}$ & $\begin{array}{c}\text { MASSA } \\
\text { SMRZANTE }\end{array}$ \\
\hline 1 & A1 & B1 & Com ângulo & Atual & Não Passante & Poliacetal & Sem \\
\hline 2 & A1 & B1 & Reto & Modificada & Não Passante & Polietileno & Com \\
\hline 3 & A1 & B2 & Com ângulo & Modificada & Passante & Poliacetal & Com \\
\hline 4 & A1 & B2 & Reto & Atual & Passante & Polietileno & Sem \\
\hline 5 & A2 & B2 & Reto & Modificada & Não Passante & Poliacetal & Sem \\
\hline 7 & A2 & B2 & Com ângulo & Atual & Não Passante & Polietileno & Com \\
\hline 8 & A2 & B1 & Reto & Atual & Passante & Poliacetal & Com \\
\hline
\end{tabular}

Tabela 4: Dados do experimento do novo cabo de embreagem.

\begin{tabular}{|c|c|c|c|c|c|c|c|c|c|c|c|c|}
\hline \multirow{2}{*}{ CORRIDA } & \multicolumn{7}{|c|}{ CONTROLE } & Rú́Do & \multicolumn{4}{|c|}{ SINAL } \\
\hline & $\mathbf{A}$ & B & c & D & E & $F$ & G & $\mathbf{N}$ & $M=15$ & $M=30$ & $M=45$ & $\mathbf{M}=\mathbf{6 0}$ \\
\hline 1 & 1 & 1 & 1 & 1 & 1 & 1 & 1 & 1 & 11 & 23 & 35 & 48 \\
\hline 1 & 1 & 1 & 1 & 1 & 1 & 1 & 1 & -1 & 12 & 26 & 39 & 53 \\
\hline 2 & 1 & 1 & -1 & -1 & 1 & -1 & -1 & 1 & 11 & 23 & 35 & 48 \\
\hline 2 & 1 & 1 & -1 & -1 & 1 & -1 & -1 & -1 & 14 & 27 & 41 & 55 \\
\hline 3 & 1 & -1 & 1 & -1 & -1 & 1 & -1 & 1 & 10 & 21 & 33 & 45 \\
\hline 3 & 1 & -1 & 1 & -1 & -1 & 1 & -1 & -1 & 13 & 26 & 39 & 52 \\
\hline 4 & 1 & -1 & -1 & 1 & -1 & -1 & 1 & 1 & 13 & 26 & 40 & 54 \\
\hline 4 & 1 & -1 & -1 & 1 & -1 & -1 & 1 & -1 & 13 & 27 & 43 & 56 \\
\hline 5 & -1 & -1 & -1 & -1 & 1 & 1 & 1 & 1 & 11 & 22 & 35 & 47 \\
\hline 5 & -1 & -1 & -1 & -1 & 1 & 1 & 1 & -1 & 13 & 27 & 41 & 56 \\
\hline 6 & -1 & -1 & 1 & 1 & 1 & -1 & -1 & 1 & 13 & 26 & 39 & 53 \\
\hline 6 & -1 & -1 & 1 & 1 & 1 & -1 & -1 & -1 & 14 & 27 & 41 & 56 \\
\hline 7 & -1 & 1 & -1 & 1 & -1 & 1 & -1 & 1 & 12 & 25 & 37 & 50 \\
\hline 7 & -1 & 1 & -1 & 1 & -1 & 1 & -1 & -1 & 13 & 27 & 40 & 54 \\
\hline 8 & -1 & 1 & 1 & -1 & -1 & -1 & 1 & 1 & 13 & 27 & 40 & 54 \\
\hline 8 & -1 & 1 & 1 & -1 & -1 & -1 & 1 & -1 & 13 & 27 & 41 & 56 \\
\hline
\end{tabular}




\section{CLASSIFICAC̣ÃO DOS SISTEMAS SINAL RESPOSTA.}

Há dois tipos comuns de sistemas sinal-resposta: Sistemas de Múltiplos Alvos (Multiple Target Systems) e Sistemas de Medição (Measurement Systems).

Um Sistema de Múltiplos Alvos é um sistema no qual a função requer que o valor da resposta possa ser ajustado pela mudança no nível do fator sinal, como, por exemplo, o sistema de moldagem por injeção mencionado na Introdução e a situação descrita na seção "Experimento para eliminação do Ruído no Novo Cabo Comando de Embreagem".

Um Sistema de Medição é aquele utilizado para obter a estimativa de alguma quantidade de interesse para uma dada unidade ou amostra, incluindo o procedimento de amostragem, preparação da amostra, calibração e o processo de medição atual. Sistema de Medição é uma aplicação prática de calibração estatística, pois medidas obtidas de uma série de padrões são usadas para calibrar o sistema. Posteriormente, estas medidas podem ser usadas para estimar a quantidade verdadeira de interesse. Nesse caso, a quantidade verdadeira do elemento presente na amostra (ou unidade avaliada) pode ser considerada como o sinal de entrada $\mathrm{M}$, a qual é convertida pelo sistema em um valor mensurado (ou resposta) Y. A precisão com que M pode ser estimado baseado em Y é determinada pelas características da relação entre $\mathrm{M}$ e Y.

\section{MEDIDAS DE DESEMPENHO (MDs)}

As propostas de MDs para cada tipo de sistema sinalresposta são apresentadas a seguir. Dentre elas está a conhecida razão sinal-ruído (S/R) dinâmica proposta por Taguchi (1986).

\section{Medidas de Desempenho para Sistemas de Medição}

Como o objetivo de um Sistema de Medição é obter uma estimativa de alguma quantidade de interesse, é razoável que o sistema possa ser avaliado com respeito à precisão das estimativas obtidas (MILLER; WU, 1996). Mandel (1964, p. 366) desenvolveu um "critério para qualidade técnica" como um método para comparar qualidades relativas de Sistemas de Medição. Taguchi (1986) considerou a "otimização de sistemas de medição" um caso especial do critério de Mandel, baseando-se em curvas lineares de calibração, e propôs como MD a razão sinal-ruído (S/R) dinâmica apresentada a seguir, partindo da idéia de que uma boa maneira para identificar uma MD adequada é especificando uma relação sinal-resposta ideal (ou alvo) e penalizando os desvios dessa função. Suponha que a função ideal seja da forma $\mathrm{E}(Y)=\beta_{0}+\beta_{1} M$, onde $\beta_{1}$ é a inclinação alvo, e que a relação atual entre o sinal e a resposta possa ser representada por $Y=\beta_{0}+\beta_{1} M+\varepsilon$, onde $E(\varepsilon)=0, V(\varepsilon)=\sigma^{2}$. Taguchi adotou $10 \log \left(\hat{\beta}_{1}^{2} / s^{2}\right)$ como MD (razão sinal-ruído, $\mathrm{S} / \mathrm{R}$ ), a qual foi justificada pelo autor com base no fato de que a medida $\mathrm{S} / \mathrm{R}$ é o inverso da variância de estimação para $\hat{M}=\left(y_{o b s}-\beta_{0}\right) / \beta_{1}$, desde que se assuma $\beta_{0}, \beta_{1}$ e $\sigma^{2}$ como conhecidos. Uma alternativa e justificativa mais rigorosa para a razão $S / R$ é baseada na amplitude dos intervalos de Fieller para o valor verdadeiro de $\mathrm{M}$ cujo valor medido de $\mathrm{Y}$ foi $y_{0}$. A amplitude esperada deste intervalo decresce se $\hat{\beta}_{1}^{2} / \sigma^{2}$ decresce, justificando a maximização da razão $\mathrm{S} / \mathrm{R}$ dinâmica.

Essa MD é baseada na relação sinal-ruído ideal ser (a) linear e (b) robusta para fatores não controláveis, porém, deve-se considerar que nem sempre é possível identificar uma relação sinal-resposta ideal e que esta MD de Taguchi assume a existência de um fator de ajuste que age como um fator de escala, o que não aparece em muitas situações práticas.

\section{Medidas de Desempenho para Sistemas de Múltiplos Alvos}

Nos Sistemas de Múltiplos Alvos o fator sinal é usado para ajustar a função do sistema para acomodar diferentes valores alvos para a resposta. A forma da função sinalresposta não é a preocupação direta para estas aplicações, contanto que todos os valores alvos desejados possam ser resultados (Miller e Wu, 1996). Seja $M=\left(m_{a}, m_{b}\right)$ a representação do intervalo conveniente do sinal que na prática pode ser aplicado ao sistema, $\mathrm{e} \mathrm{T}$ a representação dos valores alvos (valores discretos ou um intervalo).

Uma MD nesta situação pode ser uma média ponderada de desempenho para os elementos individuais de T. Assumindo que $\mathrm{V}(\mathrm{Y})$ dado $\mathrm{E}(\mathrm{Y})=y_{t}$ é uma MD conveniente, o que seria caso se aplicasse uma função perda quadrática, então uma MD pode ser definida por:

$$
M D=\sum_{y_{t} \in \mathrm{T}} V\left(Y \mid M=m_{t}\right) w\left(y_{t}\right)
$$

para valores discretos de T, onde $m_{t}=g^{-1}\left(y_{t}\right)$ e $w\left(y_{t}\right)$ é uma função ponderada baseada na importância relativa dos vários alvos. Em alguns casos, onde não for possível fixar M para $\mathrm{E}(\mathrm{Y})=y_{t}$, a $\mathrm{MD}$ pode ser modificada substituindo $\mathrm{V}(\mathrm{Y})$ pelo mínimo alcançável do Erro Quadrado Médio (EQM).

$A$ razão $S / R$ dinâmica de Taguchi é muito utilizada em alguns segmentos da indústria para Sistemas de Múltiplos Alvos, em casos onde a relação ideal entre sinal e ruído é uma reta. Entretanto, não é apropriada para este tipo de sistema, porque maximizar $10 \log \left(\hat{\beta}_{1}^{2} / s^{2}\right)$ tem o efeito de minimizar $s^{2}$, o que é sempre desejável, e maximizar $\hat{\beta}_{1}^{2}$, o que pode conduzir a resultados não desejáveis. Para $\mathrm{M}$ fixo $\left(m_{a}, m_{b}\right)$, um valor $\left|\hat{\beta}_{1}\right|$ maior pode resultar em um intervalo largo de 
valores de Y que podem estar fora dos limites de especificação de Y. Além disso, se há erro na posição de $\mathrm{M}$, esse erro será propagado para uma maior inclinação $\left|\hat{\beta}_{\mid}\right|$, resultando novamente em uma maior variação em Y.

Quando se trata da análise de dados oriundos de experimentos nos quais o sistema é do tipo sinal-resposta, algumas das estratégias propostas na literatura são adaptações daquelas propostas para sistemas com resposta única, o que acaba levando a certo tipo de confusão na apresentação do tema. Neste trabalho são abordadas três estratégias: (1) Modelagem da Medida de Desempenho (Performance Measure Modeling-PMM), também conhecida por Modelo de Perda (Loss Model); (2) Modelagem da Resposta (Response Modeling - RM) e (3) Modelagem da Função Resposta (Response Function Modeling - RFM). Estas abordagens serão descritas na próxima seção. A comparação dos resultados de cada uma delas aplicadas à situação descrita em "Experimento para eliminação do ruído no novo cabo comando de embreagem" é o principal objetivo deste trabalho.

\section{MODELAGEM E ANÁLISE DE SISTEMAS SINAL- RESPOSTA: AS ABORDAGENS PMM, RM E RFM}

Suponha que o verdadeiro modelo da resposta (Y) é linear com respeito a: (i) os $p$ fatores de controle $\left(\mathrm{C}_{1}, \mathrm{C}_{2, \ldots, \ldots} \mathrm{C}_{p}\right)$; e (ii) os $q$ fatores de ruído $\left(\mathrm{N}_{1}, \mathrm{~N}_{2}, \ldots, \mathrm{N}_{q}\right)$. Além disso, cada fator de controle $C_{l}$ interage com cada fator de ruído $N_{h}$ e com o fator de sinal $M$. Portanto, suponha que o modelo da resposta Y é dado por:

$$
\begin{aligned}
& Y=\alpha_{0}+\left(\alpha_{1} C_{1}+\ldots+\alpha_{p} C_{p}\right)+\left(\beta_{0} M+\beta_{1} M C_{1}+\ldots+\beta_{p} M C_{p}\right) \\
& +\left(\gamma_{10} N_{1}+\gamma_{11} C_{1} N_{1}+\gamma_{12} C_{2} N_{1}+\ldots+\gamma_{1 p} C_{p} N_{1}\right)+\ldots+ \\
& \left(\gamma_{q 0} N_{q}+\gamma_{q 1} C_{1} N_{q}+\gamma_{q 2} C_{2} N_{q}+\ldots+\gamma_{q p} C_{p} N_{q}\right)+\varepsilon
\end{aligned}
$$

ou de maneira equivalente:

$$
Y=\left(\alpha_{0}+\boldsymbol{\alpha}^{T} C\right)+\left(\beta_{0}+\boldsymbol{\beta}^{T} \mathbf{C}\right) M+\sum_{h=1}^{q}\left(\gamma_{h 0}+\gamma_{h}^{T} \mathbf{C}\right) N_{h}+\varepsilon
$$

onde $\mathbf{C}=\left(\mathrm{C}_{1}, \mathrm{C}_{2, \ldots,} \mathrm{C}_{p}\right)^{T}, \boldsymbol{\alpha}=\left(\alpha_{1}, \alpha_{2, \ldots,} \alpha_{p}\right)^{T}, \boldsymbol{\beta}=\left(\beta_{1}, \beta_{2, \ldots,} \beta_{p}\right)^{T}$, $\gamma_{h}=\left(\gamma_{h_{1}}, \gamma_{h_{2}, \ldots,} \gamma_{h_{p}}\right)^{T}$, os $N_{h} \mathrm{~s}$ são independentes, distribuídos segundo uma distribuição Normal (Gaussiana) com média zero e variância $\sigma_{N_{h}}^{2}, \varepsilon$ distribuído segundo uma Normal com média zero e variância $\sigma_{\varepsilon}^{2}, N_{h} \mathrm{~s}$ e $\varepsilon$ s são independentes.

Para algum valor fixo de $\mathbf{C}$, o modelo da resposta dado por (1) pode ser expresso como um modelo de regressão linear simples em $M$, isto é:

$$
Y=\alpha^{\prime}(\mathbf{C})+\beta^{\prime}(\mathbf{C}) M+e(\mathbf{C}, \mathbf{N}, \varepsilon)
$$

onde $\alpha^{\prime}(\mathbf{C})=\left(\alpha_{0}+\boldsymbol{\alpha}^{T} \mathbf{C}\right), \beta^{\prime}(\mathbf{C})=\left(\beta_{0}+\boldsymbol{\beta}^{T} \mathbf{C}\right) \mathrm{e}$

$e(\mathbf{C}, \mathbf{N}, \varepsilon)=\sum_{h=1}^{q}\left(\gamma_{h 0}+\gamma_{h}^{T} \mathbf{C}\right) N_{h}$.
As quantidades são independentes e identicamente distribuídas com média zero e variância dada por:

$$
\sigma_{e}^{2}(\mathbf{C})=\operatorname{var}(e(\mathbf{C}, \mathbf{N}, \varepsilon))=\sum_{h=1}^{q}\left(\gamma_{h 0}+\gamma_{h}^{T} \mathbf{C}\right)^{2} \sigma_{N_{h}}^{2}+\sigma_{\varepsilon}^{2}
$$

O objetivo de um experimento RPD é identificar a maneira pela qual o desempenho do sistema é afetado pelos fatores de controle e encontrar a combinação que otimiza o sistema e faz com que seu desempenho seja o menos afetado possível pelos fatores de ruído. Para alcançar este objetivo, três abordagens são apresentadas a seguir.

\section{Modelagem da Medida de Desempenho (Performance Measure Modeling - PMM) ou Modelo de Perda (Loss Model)}

$\mathrm{Na}$ abordagem PMM, medidas de desempenho (MDs) são obtidas para cada combinação dos níveis dos fatores de controle, através do ajuste do modelo dado por (2) para os dados experimentais obtidos para cada linha do arranjo-controle (cada combinação de níveis dos fatores de controle $\mathbf{C}$ presentes na matriz). As observações obtidas para uma mesma linha, porém em níveis distintos do fator de ruído, são consideradas replicações dessa condição experimental. As estimativas dos parâmetros intercepto, inclinação e variância, obtidas com o ajuste das equações (2) e (3) (ou seja, as estimativas de $\alpha^{\prime}(\mathbf{C}), \beta^{\prime}(\mathbf{C})$ e $\sigma_{\mathrm{e}}^{2}(\mathbf{C})$ ) são as MDs que são utilizadas como respostas separadas para identificar os fatores de controle com efeitos significantes para cada uma delas e determinar a condição que otimiza o sistema. Em outras palavras, um modelo linear com base no arranjo-controle é ajustado utilizando cada uma das MDs como variável resposta. Esta abordagem foi proposta por Taguchi (1986) - mas não com esta denominação - baseada na avaliação do desempenho do sistema através da razão sinal-ruído dada por $10 \log \left(\hat{\beta}_{1}^{2} / s^{2}\right)$. No caso de sistemas com resposta única, Shoemaker, Tsui e Wu (1991) classificaram este procedimento de Taguchi como Modelo de Perda (Loss Model). Por isso o termo passou a ser utilizado também para o caso de sistemas sinalresposta. Como essa abordagem usa uma determinada MD, no caso a razão $\mathrm{S} / \mathrm{R}$ passou a ter também a denominação de Modelagem da Medida de Desempenho (Performance Measure Modeling - PMM). Entretanto, outras MDs podem ser utilizadas, pois o termo $\mathrm{PMM}$ se refere à utilização de MDs em geral, sendo a razão $\mathrm{S} / \mathrm{R}$ um caso particular. Note que a variância $\sigma_{\mathrm{e}}^{2}(\mathbf{C})$ em (3) é quadrática em $\mathbf{C}$ enquanto que no modelo da resposta postulado em (1) é linear, o que pode acarretar um problema de vício na estimação no caso da abordagem PMM.

Esse procedimento pode ser explicitado matematicamente da seguinte forma. Suponha que a matriz de planejamento $\mathbf{X}_{C}$ para os fatores de controle seja representada por: 


$$
\mathbf{X}_{\mathbf{C}}=\left(\begin{array}{cccc}
1 & x_{C_{11}} & \cdots & x_{C_{p l}} \\
\cdot & \cdot & \cdots & \cdot \\
\cdot & \cdot & \cdots & \cdot \\
1 & x_{C_{1 m}} & \cdots & x_{C_{p m}}
\end{array}\right)
$$

e seja

$$
\mathbf{X}_{\mathrm{M}}=\left(\begin{array}{cc}
\mathbf{1} & \mathbf{x}_{\mathrm{M}} \\
\cdot & \cdot \\
\cdot & \cdot \\
\mathbf{1} & \mathbf{x}_{\mathrm{M}}
\end{array}\right)
$$

onde $m$ e $n$ representam a quantidade de combinações de fatores de controle e de combinações dos fatores de ruído, respectivamente, no experimento, 1 é um vetor unitário $k \times 1$, e $\mathbf{X}_{\mathrm{M}}=\left(x_{m 1}, \ldots, x_{m k}\right)^{T}$ é o vetor de $k$ valores testados do fator de sinal M. Note que $\mathbf{X}_{\mathrm{M}}$ é uma matriz de regressão $n k \times 2$ para a abordagem PMM .

Seja $\mathbf{y}_{\mathbf{i}} \mathbf{o}$ vetor resposta $n k \times 1$ correspondente à i-ésima linha da matriz de planejamento dos fatores de controle $\mathbf{X}_{\mathrm{C}}$. De acordo com a abordagem PMM, para cada linha de $\mathbf{X}_{\mathrm{C}}$, um modelo de regressão linear simples é ajustado para $\mathbf{y}_{\mathbf{i}}$, e as estimativas de intercepto, inclinação e variância são calculadas como a seguir. Denote por $\mathbf{b}_{\mathbf{i}}$ o vetor $2 \times 1$ das estimativas de regressão para o intercepto e a inclinação:

$\mathbf{b}_{\mathbf{i}}=\left(\mathbf{X}_{\mathbf{M}}^{\mathbf{T}} \mathbf{X}_{\mathbf{M}}\right)^{-1}\left(\mathbf{X}_{\mathbf{M}}^{\mathbf{T}} \mathbf{y}_{\mathbf{i}}\right)$ para $i=1,2, \ldots, m$.

De maneira similar, a estimativa de $\sigma_{\mathrm{e}}^{2}(\mathbf{C})$ em (3) é dada por:

$$
S_{i}^{2}=\left(\mathbf{y}_{\mathbf{i}}-\mathbf{X}_{\mathbf{M}} \mathbf{b}_{\mathbf{i}}\right)^{\mathbf{T}}\left(\mathbf{y}_{\mathbf{i}}-\mathbf{X}_{\mathbf{M}} \mathbf{b}_{\mathbf{i}}\right) /(n k-2) \text {, para } i=1, \ldots, m .
$$

Depois de ajustar os modelos de regressão linear simples, o próximo passo é tratar cada estimativa de regressão como uma resposta e identificar os efeitos dos fatores de controle que afetam as estimativas de regressão. Como a matriz de planejamento dos fatores de controle $\mathbf{X}_{\mathrm{C}}$ é ortogonal, os efeitos dos fatores de controle podem ser estimados por:

$$
\hat{\boldsymbol{\Theta}}_{\mathbf{b}}=\mathbf{X}_{\mathbf{C}}^{\mathbf{T}} \mathbf{b} / m
$$

onde $\mathbf{b}=\left(\mathbf{b}_{1}, \mathbf{b}_{2}, \ldots, \mathbf{b}_{\mathrm{m}}\right)^{\mathrm{T}}$ e $\hat{\boldsymbol{\Theta}}_{\mathbf{b}}$ é uma matriz $(\mathrm{p}+1) \times 2$ onde as duas colunas representam as estimativas dos fatores de controle que afetam o intercepto e a inclinação, respectivamente. De maneira similar,

$\hat{\boldsymbol{\Theta}}_{\mathrm{S}}=\mathbf{X}_{\mathrm{C}}^{\mathbf{T}} \mathbf{S}^{2} / m$,

onde $\mathbf{S}^{2}=\left(S_{1}^{2}, \ldots, S_{m}^{2}\right)^{\mathrm{T}}$ e $\hat{\boldsymbol{\Theta}}_{\mathbf{S}}$ representam os efeitos das estimativas dos fatores de controle que afetam a variância $\sigma_{\text {e }}^{2}$.
$\mathrm{Na}$ abordagem PMM, o objetivo é determinar as combinações dos fatores de controle baseadas nessas estimativas que minimizam a variância do processo enquanto mantêm o intercepto e a inclinação nos seus valores alvos.

\section{Modelagem da Resposta (Response Modeling - RM]}

$\mathrm{Na}$ abordagem $\mathrm{RM}$, a idéia é primeiro estimar todos os parâmetros da equação geral para a resposta $Y$ (1). Para isso, usam-se como matriz para ajuste do modelo o arranjo combinado (matriz contendo tanto os fatores de controle como de ruído, bem como os valores do sinal $\mathrm{M}$ ) e os dados brutos originais. $\mathrm{O}$ foco é, portanto, modelar a resposta $\mathrm{Y}$ em função de $\mathbf{C}, \mathbf{N}$ e $\mathrm{M}$ e não a relação entre $\mathrm{Y}$ e $\mathbf{M}$.

Assim, seja $\mathbf{X}_{N}$ a matriz de planejamento $n \times q$ dos fatores de ruído, ortogonal à matriz $\mathbf{X}_{\mathrm{C}}$ dos fatores de controle, dada por:

$\mathbf{X}_{\mathbf{N}}=\left(\begin{array}{ccc}x_{N_{11}} & \cdots & x_{N_{\mathrm{q} 1}} \\ \cdot & \cdots & \cdot \\ \cdot & \cdots & \cdot \\ x_{N_{1 n}} & \ldots & x_{N_{\mathrm{qn}}}\end{array}\right)$

Seja ainda $\mathbf{X}_{\mathrm{NK}}$ uma matriz $n k \times q$ construída a partir de $\mathbf{X}_{N}$ definida como:

$$
\mathbf{X}_{\mathrm{NK}}=\left(\begin{array}{ccc}
x_{N_{11}} \mathbf{1} & \ldots & x_{N q l} \mathbf{1} \\
\cdot & \ldots & \cdot \\
\cdot & \ldots & \cdot \\
x_{N_{1 n}} \mathbf{1} & \ldots & x_{N q n} \mathbf{1}
\end{array}\right)
$$

onde 1 é um vetor unitário $k \times 1$.

$\mathrm{Na}$ abordagem $\mathrm{RM}$, é preciso primeiramente determinar a matriz de planejamento para o modelo geral (1). Portanto a matriz de regressão do modelo (1) de dimensão $m n k x$ $(2+p+q+p q)$ pode ser escrita da seguinte forma:

$\mathbf{X}=\left(\begin{array}{cccccccccc}\mathbf{X}_{\mathrm{M}} & x_{c_{11}} \mathbf{X}_{\mathrm{M}} & \ldots & x_{p 1} \mathbf{X}_{\mathrm{M}} & \mathbf{X}_{\mathrm{NK}} & x_{c_{11}} \mathbf{X}_{\mathrm{NK}} & \ldots & x_{C_{1 p}} \mathbf{X}_{\mathrm{NK}} \\ \cdot & \cdot & \ldots & \cdot & \cdot & \cdot & \ldots & \cdot \\ \cdot & \cdot & \ldots & \cdot & \cdot & \cdot & \ldots & \cdot \\ \mathbf{X}_{\mathrm{M}} & x_{c_{1 m}} \mathbf{X}_{\mathrm{M}} & \ldots & x_{p m} \mathbf{X}_{\mathrm{M}} & \mathbf{X}_{\mathrm{NK}} & x_{C m} \mathbf{X}_{\mathrm{NK}} & \ldots & x_{C p m} \mathbf{X}_{\mathrm{NK}}\end{array}\right)(5)$

As $(2+2 p+q+p q)$ colunas acima correspondem aos seguintes parâmetros no modelo $(1):\left(\alpha_{0}, \beta_{0}\right),\left(\alpha_{1}, \beta_{1}\right), \ldots,\left(\alpha_{p}, \beta_{p}\right)$, $\left(\gamma_{10}, \gamma_{q 0}\right),\left(\gamma_{11}, \gamma_{q 1}\right), \ldots,\left(\gamma_{1 p}, \gamma_{q p}\right)$. Os blocos das colunas em (5) são ortogonais, assim como as colunas nas matrizes $\mathbf{X}_{\mathrm{C}} \mathrm{e}$ $\mathbf{X}_{\mathrm{N}}$ também são. Logo, a matriz $\mathbf{X}^{\mathrm{T}} \mathbf{X}$ é uma matriz diagonal blocada, isto é, 


$\mathbf{X}^{\mathrm{T}} \mathbf{X}=m\left(\begin{array}{cccccccc}\mathbf{X}_{\mathbf{M}}^{\mathrm{T}} \mathbf{X}_{\mathbf{M}} & 0 & \ldots & . & \cdot & \cdot & \ldots & 0 \\ 0 & \mathbf{X}_{\mathbf{M}}^{\mathrm{T}} \mathbf{X}_{\mathbf{M}} & \ldots & . & . & . & \ldots & 0 \\ . & . & \mathbf{X}_{\mathrm{M}}^{\mathrm{T}} \mathbf{X}_{\mathrm{M}} & . & . & . & \ldots & . \\ 0 & 0 & \ldots & \mathbf{X}_{\mathrm{M}}^{\mathrm{T}} \mathbf{X}_{\mathrm{M}} & 0 & 0 & \ldots & 0 \\ 0 & 0 & \ldots & 0 & (n k) \mathbf{I q} & 0 & \ldots & 0 \\ 0 & 0 & \ldots & 0 & 0 & (n k) \mathbf{I q} & \ldots & 0 \\ . & . & \ldots & . & . & . & \ldots & . \\ 0 & 0 & \ldots & 0 & 0 & 0 & \ldots & (n k) \mathbf{I q}\end{array}\right)$

tante independente de C. Neste caso, a variância do processo, $\sigma_{\mathrm{e}}^{2}$, pode ser estimada ignorando $\sigma_{\varepsilon}^{2}$, e o resultado ótimo não será afetado. Contudo, no caso em que $\sigma_{\varepsilon}^{2}$ é uma função de $\mathbf{C}$, tem que primeiro ser estimada com base no modelo de resposta estimado. Então, os efeitos dos fatores de controle que afetam as estimativas da variância do erro puro serão identificados e esta informação será usada para determinar as combinações ótimas dos fatores de controle.

onde $\mathbf{I}_{\mathbf{q}}$ é uma matriz identidade $q$-dimensional, e o inverso da matriz $\mathbf{X}^{\mathrm{T}} \mathbf{X}$ também é uma matriz diagonal blocada. De maneira similar, se $\mathbf{y}^{\mathrm{T}}=\left(\mathbf{y}_{1}^{\mathrm{T}}, \ldots, \mathbf{y}_{\mathrm{m}}^{\mathrm{T}}\right)$, a matriz $\mathbf{X}^{\mathrm{T}} \mathbf{y}$ pode ser expressa como:

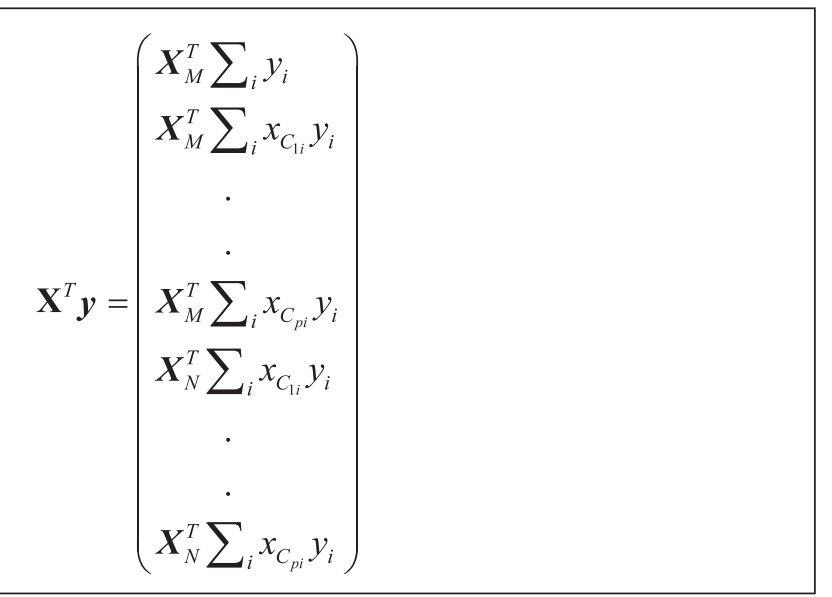

Segue que os parâmetros estimados do modelo (1) $\hat{\boldsymbol{\Theta}}_{\mathbf{R}}=\left(\left(\hat{\alpha}_{0}, \hat{\beta}_{0}\right),\left(\hat{\alpha}_{1}, \hat{\beta}_{1}\right), \ldots,\left(\hat{\alpha}_{p}, \hat{\boldsymbol{\beta}}_{p}\right),\left(\hat{\gamma}_{10}, \ldots, \hat{\gamma}_{q 0}\right),\left(\hat{\gamma}_{11}, \ldots, \hat{\gamma}_{q 1}\right), \ldots\right.$, $\left.\left(\hat{\gamma}_{1 p}, \ldots, \hat{\gamma}_{q p}\right)\right)^{\mathrm{T}}$, podem ser calculados da seguinte maneira:

\begin{tabular}{|c|c|c|}
\hline$\hat{\mathbf{\Theta}}_{\mathbf{R}}=\left(\mathbf{X}^{\mathbf{T}} \mathbf{X}\right)^{-1}\left(\mathbf{X}^{\mathbf{T}} \mathbf{y}\right)=m^{-1}$ & $\left.\begin{array}{c}\sum_{i}\left(\mathbf{X}_{\mathbf{M}}^{\mathrm{T}} \mathbf{X}_{\mathbf{M}}\right)^{-1}\left(\mathbf{X}_{\mathbf{M}}^{\mathrm{T}} y_{i}\right) \\
\sum_{i} x_{c_{1 i}}\left(\mathbf{X}_{\mathbf{M}}^{\mathrm{T}} \mathbf{X}_{\mathbf{M}}\right)^{-1}\left(\mathbf{X}_{\mathbf{M}}^{\mathrm{T}} y_{i}\right) \\
\cdot \\
\sum_{i} x_{c_{p i}}\left(\mathbf{X}_{\mathbf{M}}^{\mathrm{T}} \mathbf{X}_{\mathbf{M}}\right)^{-1}\left(\mathbf{X}_{\mathbf{M}}^{\mathrm{T}} y_{i}\right) \\
(n k)^{-1} \sum_{i} \mathbf{X}_{\mathbf{N K}}^{\mathbf{T}} y_{i} \\
(n k)^{-1} \sum_{i} x_{C_{1 i}} \mathbf{X}_{\mathbf{N K}}^{\mathbf{T}} y_{i} \\
\cdot \\
\cdot \\
(n k)^{-1} \sum_{i} x_{C_{p i}} \mathbf{X}_{\mathbf{N K}}^{\mathbf{T}} y_{i}\end{array}\right)=$ & $\left(\begin{array}{c}\left(\mathbf{X}_{\mathrm{C}}^{\mathrm{T}} \mathbf{b}\right) / m \\
(m n k)^{-1} \mathbf{X}_{\mathrm{NK}}^{\mathrm{T}} \sum_{i} y_{i} \\
(m n k)^{-1} \mathbf{X}_{\mathbf{N K}}^{\mathbf{T}} \sum_{i} x_{C_{1 i}} y_{i} \\
\cdot \\
\cdot \\
(m n k)^{-1} \mathbf{X}_{\mathbf{N K}}^{\mathbf{T}} \sum_{i} x_{C_{p i}} y_{i}\end{array}\right)$ \\
\hline
\end{tabular}

\section{Comparação das abordagens PMM e RM}

Comparando as equações (4) e (6), observa-se que os efeitos estimados $\left(\hat{\boldsymbol{\Theta}}_{\mathbf{b}}\right)$ para as funções intercepto e inclinação na abordagem PMM são idênticos aos da abordagem RM, isto é, a primeira parte dos efeitos esti$\operatorname{mados}\left(\hat{\boldsymbol{\Theta}}_{\mathrm{R}}\right)$ em $(6),\left(\hat{\alpha}_{0}, \hat{\beta}_{0}\right), \ldots,\left(\hat{\alpha}_{p}, \hat{\boldsymbol{\beta}}_{p}\right)$. Isto ocorre por que os procedimentos de estimação em ambos os métodos são lineares e os efeitos dos fatores de controle que afetam essas funções em (2) são os mesmos parâmetros no modelo de resposta original (1). Porém, o mesmo não ocorre para a função variância em (3) por ser uma função quadrática dos parâmetros em (1). Além de ter estimativas mais confiáveis para os efeitos dos fatores de controle, o método RM tem outra vantagem sobre o método PMM: providencia informação adicional sobre como os fatores de ruído individualmente reduzem a variância do processo, o que não é fornecido pelo método PMM.

\section{Modelagem da Função Resposta (Response Function Modeling - RFM)}

$\mathrm{Na}$ abordagem RFM, o primeiro passo é modelar a relação sinal-resposta (isto é, a relação entre Y e M) através de um modelo da forma (2). Este modelo é ajustado aos dados experimentais brutos, oriundos de cada combinação de níveis dos fatores $\mathrm{C}$ e N. Difere da abordagem PMM nesta etapa, pois aqui as observações oriundas de uma mesma combinação de níveis dos fatores de controle em diferentes níveis de $\mathrm{N}$ não são consideradas replicações. Neste caso, o modelo (2) pode ser reescrito como:

$$
Y \mid(C, N)=\tilde{\alpha}(C, N)+\widetilde{\beta}(C, N) M+\tilde{e}(C, N, \varepsilon)
$$

indicando que os parâmetros do modelo agora podem depender tanto dos fatores de controle como dos fatores de ruído. $\mathrm{O}$ segundo passo é usar as estimativas dos parâmetros $\widetilde{\alpha}(C, N), \widetilde{\beta}(C, N)$ e $\widetilde{\sigma}_{\mathrm{e}}^{2}(C, N)$ como respostas e identificar o set-up dos fatores de controle que otimizam o sistema. Em outras palavras, um modelo linear com base no arranjo combinado (controle + ruído) é ajustado utilizando cada uma destas estimativas como variável resposta. Estas três abordagens serão utilizadas na 
situação descrita na seção "Experimento para eliminação do ruído no novo cabo comando de embreagem".

\section{ANÁLISE DOS DADOS DO EXPERIMENTO NO CABO COMANDO DE EMBREAGEM}

Os dados do experimento foram analisados por Borges e Freitas (2005) utilizando a razão sinal-ruído (S/R) dinâmica de Taguchi. Nesta seção, os dados serão reanalisados com base nas três abordagens apresentadas na Seção "Modelagem e análise de sistemas sinal-resposta: as abordagens PMM, RM e RFM". Todas as análises foram implementadas no software Minitab ${ }^{\circ}$, versão 14 .

\section{Modelagem da Medida de Desempenho - PMM}

Um modelo de regressão linear simples foi ajustado, pelo método de mínimos quadrados, para cada uma das oito configurações possíveis ilustradas na Tabela 3 e cujos dados estão na Tabela 4. Neste caso, para efeitos de ajuste do modelo, os resultados obtidos para uma mesma configuração, porém em níveis diferentes do fator de ruído N, foram considerados replicações. Portanto, oito estimativas de intercepto $\left(\hat{\beta}_{0}\right)$, inclinação $\left(\hat{\beta}_{1}\right)$ e variância do erro $\left(\hat{\sigma}^{2}\right)$ foram obtidas. Estes resultados estão na Tabela 5 .

Assim, após obter as estimativas das MDs (intercepto, inclinação e variância), o próximo passo foi utilizá-las como resposta e obter estimativas dos efeitos dos fatores de con-

Tabela 5: Estimativas de $\hat{\beta}_{0}, \hat{\beta}_{1}$ e $\hat{\sigma}^{2}$ pela análise PMM do experimento do novo cabo de embreagem.

\begin{tabular}{|c|c|c|c|}
\hline CONFIGURAÇÄO & $\hat{\beta}_{0}$ & $\hat{\beta}_{1}$ & $\hat{\sigma}^{2}$ \\
\hline 1 & $-1,50$ & 0,863 & 4,31 \\
\hline 2 & $-0,75$ & 0,867 & 9,248 \\
\hline 3 & $-1,00$ & 0,823 & 9,941 \\
\hline 4 & $-1,25$ & 0,940 & 1,316 \\
\hline 5 & $-1,50$ & 0,880 & 12,264 \\
\hline 6 & $-0,50$ & 0,910 & 1,442 \\
\hline 7 & $-0,50$ & 0,873 & 2,566 \\
\hline 8 & $-1,00$ & 0,930 & 0,475 \\
\hline
\end{tabular}

Tabela 6: Estimativas dos coeficientes associados aos efeitos dos fatores de controle sobre as MDs, $\hat{\beta}_{0}, \hat{\beta}_{1}$ e $\hat{\sigma}^{2}$, pela abordagem PMM.

\begin{tabular}{|c|c|c|c|}
\hline EFEITO & $\hat{\beta}_{0}$ & $\hat{\beta}_{1}$ & $\hat{\sigma}^{2}$ \\
\hline constante & $-1,000$ & 0,886 & 5,195 \\
\hline A - Fornecedor da Boccola & $-0,125$ & $-0,0013$ & 1,008 \\
\hline B - Fornecedor do Tasselo & 0,063 & $-0,003$ & 1,046 \\
\hline C - Terminal & 0,000 & $-0,004$ & $-1,153$ \\
\hline D - Layout & 0,063 & 0,011 & $-2,787$ \\
\hline E - Tubetto & $-0,063$ & $-0,006$ & 1,621 \\
\hline F - Material do tubetto & $-0,125$ & $-0,026$ & 2,075 \\
\hline G - Massa Smorzante & $-0,313$ & 0,018 & $-0,604$ \\
\hline
\end{tabular}


trole sobre cada uma delas. A Tabela 6 mostra as estimativas dos coeficientes associados a estes efeitos.

As Figuras 1 a 3 mostram os gráficos de probabilidade normal das estimativas dos efeitos obtidos para as análises realizadas para cada uma das MDs. O método de Lenth (1989) foi usado para identificar os efeitos significativos. Ao nível de $10 \%$ de significância, os fatores de controle com efeitos não significativos foram sendo eliminados, até chegar aos modelos abaixo, incluindo apenas os significativos:

$$
\hat{\beta}_{0}=-1,000-0,125 A-0,125 F-0,313 G
$$

$\hat{\beta}_{1}=0,886-0,013 A+0,011 D-0,026 F+0,018 G$

$$
\hat{\sigma}^{2}=5,195-2,787 D
$$

O fator "Layout" é o único que afeta significativamente na variância. Quando fixado no nível 1, que corresponde ao Layout Atual, reduz a variância do processo para 2,408. Os fatores "Material do Tubetto", "Massa Smorzante", "Fornecedor de Boccola" e "Layout" são os que têm influência na inclinação do sistema. Como o fator "Layout" também influencia na variância, considera-se o nível que foi fixado anteriormente. Logo, os fatores fixados nos níveis $-1,1,-1$ e 1, respectivamente, correspondem a usar o Polietileno como Material do Tubetto, Sem Massa Smorzante, Fornecedor A2 de Boccola e Layout Atual, na fabricação do cabo de embreagem. Com estes níveis fixados obtém-se o valor 0,954 para a inclinação, combinação que se aproxima mais do valor ideal, que é igual a 1. Já os fatores que interferem significativamente no intercepto, também interferem na inclinação, que são "Massa Smorzante", "Fornecedor de Boccola" e "Material do Tubetto". Sendo assim, considerando os níveis fixados para a inclinação, obtém-se o valor 0,937 para o intercepto.

\section{Modelagem da Resposta - RM}

$\mathrm{O}$ procedimento desse tipo de modelagem consiste em primeiro ajustar o modelo (1) através de análise de regressão pelo método de mínimos quadrados. A resposta é modelada em função dos fatores de controle, de ruído e sinal. Os parâmetros estimados e os valores da estatística teste t são mostrados na Tabela 7 .

Após o ajuste do modelo completo (Tabela 7), os termos não significativos ao nível de significância de $10 \%$ foram sendo excluídos do modelo até chegar ao modelo final reduzido mostrado na equação 10 , composto apenas por termos significativos e que apresentou um $\mathrm{R}^{2}=99,6 \%$. Os termos significativos são os marcados na coluna "Estatística t" da Tabela 7 por $(*)$.

$$
\begin{aligned}
& Y=-1,000+(0,886-0,013 A-0,026 F+0,018 G) M+ \\
& (-1,594-0,281 A+0,219 C+0,469 D-0,344 E-0,469 F \\
& +0,219 G) N
\end{aligned}
$$

Como foi dito na seção "Comparação das abordagens

Figura 1: Gráfico de probabilidade normal para as estimativas dos efeitos para $\hat{\beta}_{0}$ (abordagem PMM)

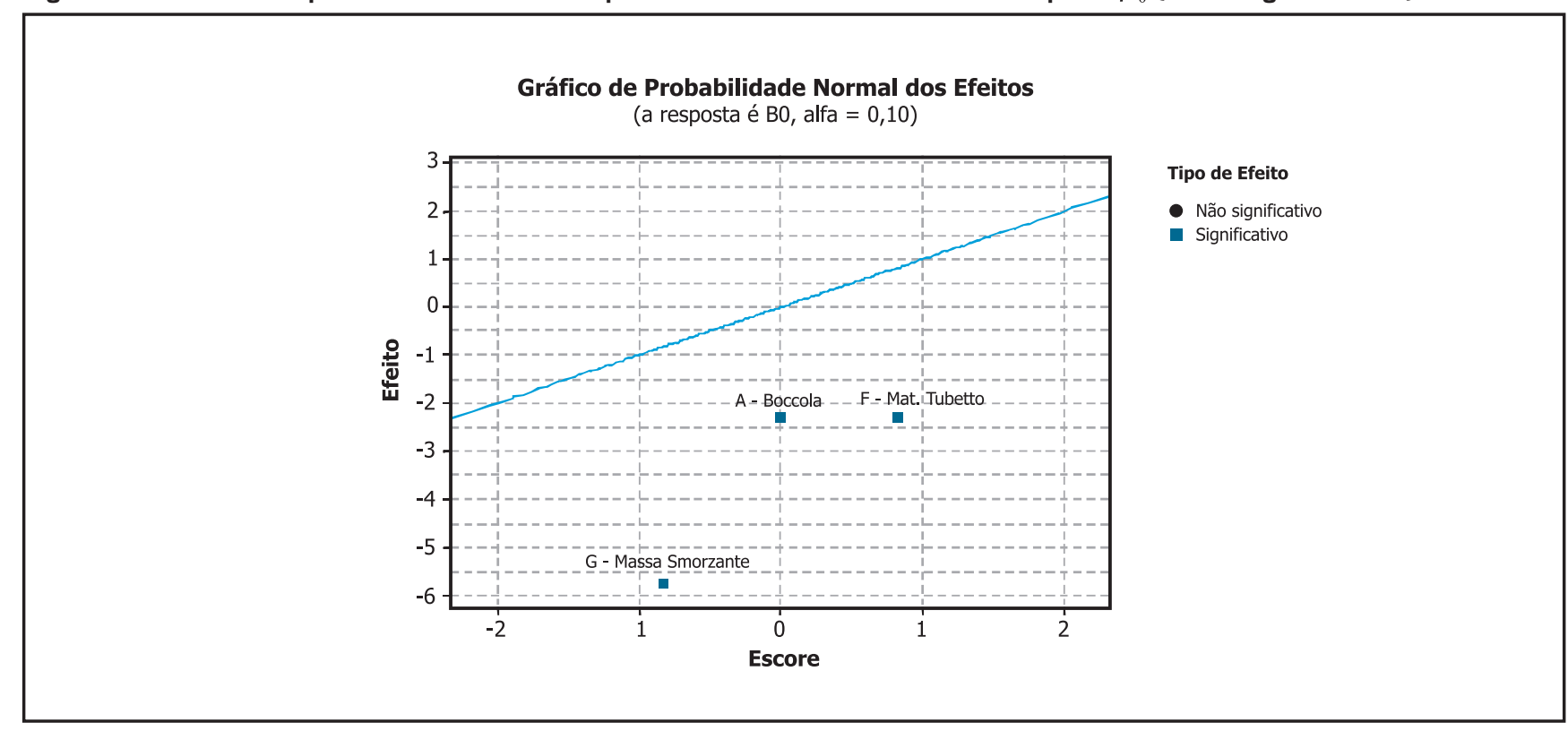


PMM e RM", as estimativas dos efeitos dos fatores de controle das funções intercepto e inclinação no método RM são idênticas às estimativas do modelo PMM. Essa igualdade pode ser visualizada comparando as estimativas dos efeitos sobre $\beta_{0}$ do método RM (estimativas dos parâmetros dos efeitos principais para $A, B, \ldots, G$ da Tabela 7) com as estimativas para os efeitos sobre $\beta_{0}$ do método PMM na coluna 2 da Tabela 6 . Também, quando se comparam as estimativas dos efeitos sobre $\beta_{1}$ do método RM (estimativas dos parâmetros das interações $A M, B M, \ldots, G M$ da Tabela 7) com as estimativas para os efeitos sobre $\beta_{1}$ do método PMM na coluna 3 da Tabela 6 .

Para estimar os efeitos dos fatores de controle sobre a variância do processo, o método é baseado em Box e Jones (1990). Primeiro, estima-se a variância causada pelos fatores de ruído externos. Assumindo que a variável de ruído $\mathrm{N}$ em

Figura 2: Gráfico de probabilidade normal para as estimativas dos efeitos para $\hat{\beta}_{1}$ (abordagem PMM).

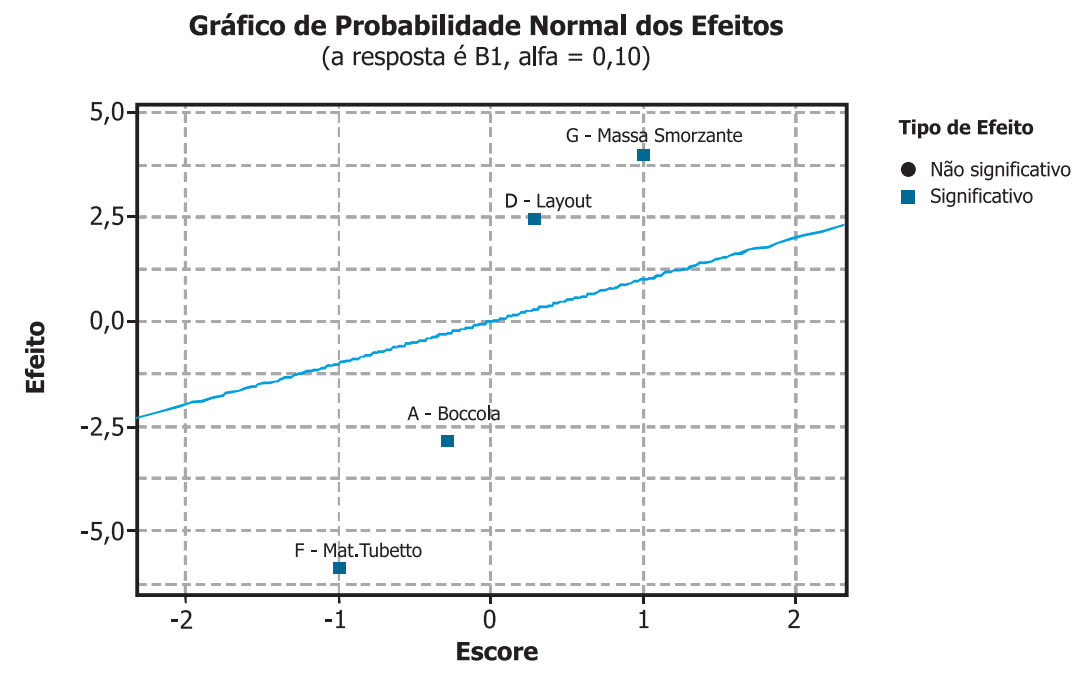

Figura 3: Gráfico de probabilidade normal para as estimativas dos efeitos para $\hat{\sigma}^{2}$ (abordagem PMM).

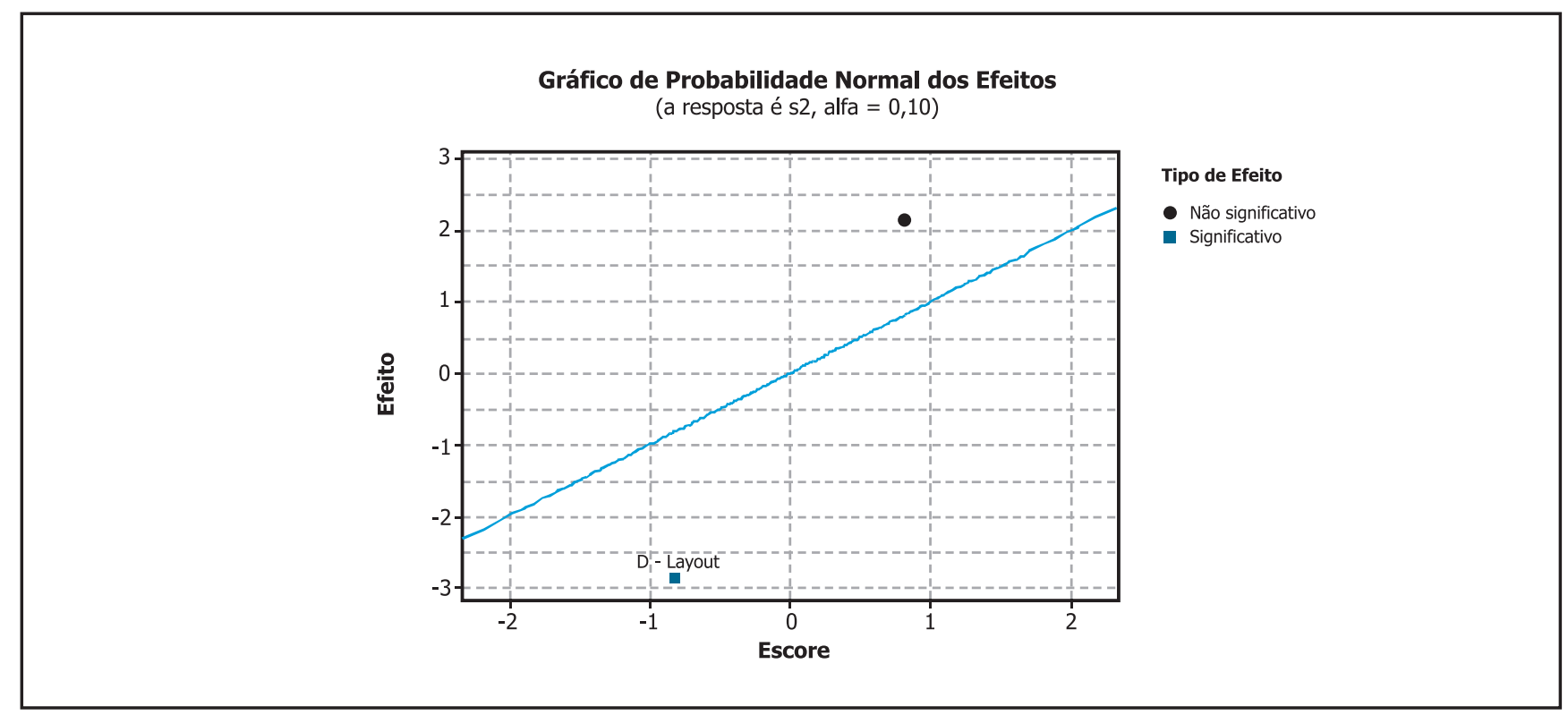


(10) é aleatória com variância igual a um, a variância de $\hat{y}$ sobre os fatores de ruído externo é estimada por:

$$
\begin{aligned}
& \operatorname{Var}(\hat{y} \mid N)=(-1,594-0,281 A+0,219 C+0,469 D \\
& -0,344 E-0,469 F+0,219 G)^{2}
\end{aligned}
$$

que é uma função quadrática dos fatores $A, C, D, E, F \mathrm{e}$ $G$. Para avaliar se a variância erro-puro $\hat{\sigma}_{e p}^{2}$ é afetada pelos fatores de controle, os resíduos do modelo ajustado (10) são calculados. Depois, uma amostra da variância sobre as combinações de ruído e sinal é obtida para cada com- binação de fatores de controle (corrida) e essas variâncias amostrais representam as estimativas de $\hat{\sigma}_{e p}^{2}$. Para identificar quais fatores de controle tinham efeito significativo sobre $\hat{\sigma}_{e p}^{2}$, utilizou-se o gráfico de probabilidade normal pelo método de Lenth (1989). Ao nível de significância de $10 \%$, visualiza-se na Figura 4 que nenhum fator foi significativo.

Segue então que a variância do processo $\hat{\sigma}^{2}$ é igual à variância devida aos fatores de ruído, já que para a variância devida ao erro-puro não houve fator significativo, logo a mesma é considerada nula:

Tabela 7: Estimativas dos coeficientes associados aos efeitos sobre a resposta para o modelo completo ajustado

\begin{tabular}{|c|c|c|}
\hline EFEITO & ESTIMATIVAS & ESTATÍSTICA T \\
\hline Constante & $-1,000$ & $-3,33[*]$ \\
\hline A - Fornecedor da Boccola & $-0,125$ & $-0,42$ \\
\hline B - Fornecedor do Tasselo & 0,063 & 0,21 \\
\hline C - Terminal & 0,000 & 0,00 \\
\hline D - Layout & 0,063 & 0,21 \\
\hline E - Tubetto & $-0,063$ & $-0,21$ \\
\hline F - Material do Tubetto & 0,125 & $-0,42$ \\
\hline G - Massa Smorzante & $-0,313$ & $-1,04$ \\
\hline M - Sinal & 0,886 & 121,30 [*] \\
\hline AM - Fornecedor da Boccola*Sinal & $-0,013$ & $-1,71[*]$ \\
\hline BM - Fornecedor do Tasselo*Sinal & $-0,003$ & $-0,34$ \\
\hline CM - Terminal*Sinal & $-0,004$ & $-0,57$ \\
\hline DM - Layout*Sinal & 0,011 & 1,48 \\
\hline EM - Tubetto*Sinal & $-0,006$ & $-0,80$ \\
\hline FM - Material do Tubetto*Sinal & $-0,026$ & $-3,54[*]$ \\
\hline GM - Massa Smorzante*Sinal & 0,018 & $2,40[*]$ \\
\hline N - Ruído & $-1,594$ & $-13,01(*)$ \\
\hline AN - Fornecedor da Boccola*Ruído & $-0,281$ & $-2,30[*]$ \\
\hline BN - Fornecedor do Tasselo*Ruído & 0,156 & 1,28 \\
\hline CN - Terminal*Ruído & 0,219 & 1,79 [*] \\
\hline DN - Layout*Ruído & 0,469 & 3,83 (*) \\
\hline EN - Tubetto*Ruído & $-0,344$ & $-2,81[*]$ \\
\hline FN - Material do Tubetto*Ruído & $-0,469$ & $-3,83[*]$ \\
\hline GN - Massa Smorzante*Ruído & 0,219 & $1,79[*]$ \\
\hline
\end{tabular}
aos dados do experimento do novo cabo da embreagem - abordagem RM ( "* " indica significância a nível 10\%). 


$$
\begin{aligned}
& \hat{\sigma}^{2}=\operatorname{Var}(\hat{y} \mid N)+\hat{\sigma}_{e p}^{2} \\
& =(-1,594-0,281 A+0,219 C+0,469 D-0,344 E \\
& -0,469 F+0,219 G)^{2}
\end{aligned}
$$

Como não há interações significativas entre os fatores de controle e o fator de ruído no modelo resposta, é impossível reduzir a variância do processo usando interações controle e ruído. Para minimizar a variância do processo dentro da região experimental, mantendo a inclinação no valor alvo, fixam-se $C=-1$ e $E=-1$, o que corresponde ao Terminal Reto e Tubetto Passante.

\section{Modelagem da Função Resposta - RFM}

As estimativas das funções locação (intercepto e inclinação) e dispersão (variância) foram calculadas ajustando modelos de regressão linear pelo método de mínimos quadrados para cada uma das 16 combinações de fatores de controle e de ruído cujos dados estão na Tabela 4. Nesta modelagem, uma configuração da Tabela 4 corresponde a duas combinações distintas, já que se leva em conta o nível da condição de ruído. Como não existem réplicas das observações em cada nível do fator sinal para essas combinações, não foi possível separar a variação total em dois componentes (falta de ajuste e erropuro) e analisá-los separadamente. Os resultados dos ajustes dos modelos de regressão estão resumidos na Tabela 8 .

Após obter as estimativas das funções locação e dispersão, os efeitos dos fatores de controle e ruído sobre esses parâmetros foram avaliados. A Tabela 9 mostra as estimativas destes efeitos.

As Figuras 5 a 7 contêm os gráficos de probabilidade normal dos efeitos sobre cada uma das funções e o método de
Lenth (1989) foi usado para identificar os efeitos significativos ao nível de significância de $10 \%$, eliminando os fatores não significativos. Os modelos ajustados foram:

$$
\begin{aligned}
\hat{\beta}_{0}= & -1,000-0,125 A+0,062 D-0,125 F-0,313 G+ \\
& (-0,125-0,250 A+0,188 D+0,312 G) N
\end{aligned}
$$

$$
\begin{aligned}
\hat{\beta}_{1}= & 0,886-0,013 A+0,011 D-0,026 F+0,018 G+ \\
& (-0,039-0,009 F) N
\end{aligned}
$$

$\hat{\sigma}^{2}=0,200-0,075 B-0,038 C+0,050 D-0,026 F+0,038 G+$ $(0,038+0,038 B-0,075 D+0,088 F) N$

Inicialmente, considere o modelo ajustado para $\hat{\beta}_{1}$. Os fatores de controle "Fornecedor de Boccola", "Material do Tubetto" e "Massa Smorzante" são os que têm influência na inclinação do sistema. Observe que o fator de ruído "N" e as interações entre "Material do Tubetto e N" e "Layout e N" também foram significativos. Como a interação "Layout e N" foi significativa, o fator "Layout" também deve entrar no modelo. Logo, os fatores de controle "Fornecedor de Boccola", "Layout", "Material do Tubetto" e "Massa Smorzante" fixados nos níveis -1, 1, -1 e 1, respectivamente, correspondem a usar o Fornecedor A2 de Boccola, Layout Atual, Polietileno como Material do Tubetto e Sem Massa Smorzante na fabricação do cabo de embreagem. Fixando estes níveis obtém-se o valor 0,954 para a inclinação, combinação que se aproxima mais do valor ideal que é igual a 1, e que torna o sistema menos sensível a mudanças em $\mathrm{N}$.

Figura 4: Gráfico de probabilidade Normal para as estimativas de efeitos dos fatores para o erro puro.

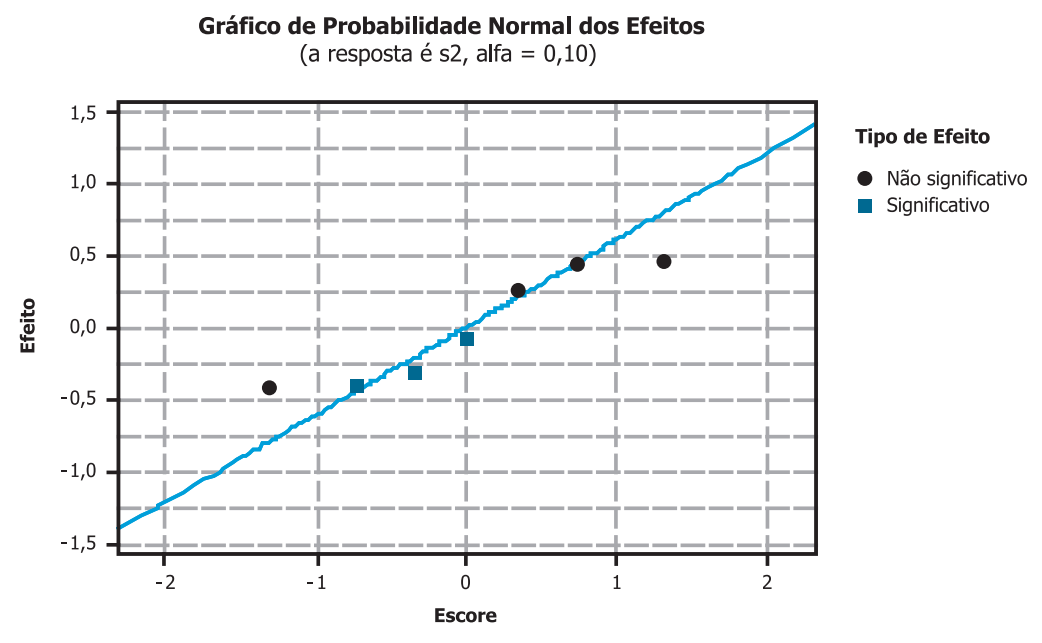


Depois, considere o modelo para $\hat{\sigma}^{2}$. São significativos os fatores de controle "Fornecedor de Tasselo", "Terminal", "Layout", "Material do Tubetto" e "Massa Smorzante". Considerando os níveis dos fatores fixados para ajustar $\hat{\beta}_{1}$ ao valor alvo, e com o objetivo de reduzir a variabilidade, os fatores de controle "Fornecedor de Tasselo" e "Terminal" devem ser fixados ambos no nível 1, o que corresponde a usar Fornecedor B1 de Tasselo e Terminal Com Ângulo. $\mathrm{O}$ fator de ruído composto "N" e as interações do fator de ruído com Fornecedor de Tasselo, Layout e Material de Tubetto, também afetam a variância. Com os níveis fixados anteriormente, tem-se que o fator de ruído $\mathrm{N}$ afeta pouco na variabilidade.

Para a função $\hat{\beta}_{0}$, tem-se que "Fornecedor de Boccola", "Material do Tubetto" e "Massa Smorzante" são os fatores de controle com efeitos significativos. Além desses fatores, o fator de ruído " $\mathrm{N}$ " e as interações do fator de ruído com Fornecedor de Boccola, Layout, Material do Tubetto e Massa Smorzante também são significativos. Como a interação entre Layout e ruído é significativa, o fator Layout também teve que ser incluído no modelo.

\section{Discussão dos Resultados}

Nota-se que os efeitos significativos e as combinações ótimas identificadas são os mesmos para as funções intercepto e inclinação para as três metodologias. As conclusões tornam-se diferentes quando se analisa a variância do processo. É claro que os modelos RFM e RM fornecem informação adicional de como a variância do processo pode ser minimizada. O método RFM permite avaliar o efeito que o fator de ruído tem sobre as funções intercepto, inclinação e variância. Com essa informação, busca-se por uma combinação ótima de forma que o ruído afete o menos possível as funções, ou pelo menos, avalia-se o quanto o ruído influencia os valores dessas funções. $\mathrm{O}$ método $\mathrm{RM}$ permitiu dividir a variância total do processo em duas, e verificar que a variância se deve aos níveis dos fatores de ruído, e não ao erro-puro. Porém, o modelo ajustado para a variância pelo método RM ficou mais confuso que os modelos dos demais métodos.

Ressalta-se que no estudo do cabo comando de embreagem foi utilizado um experimento com sete fatores e, tanto na abordagem de análise original quanto nas abordagens alternativas apresentadas neste artigo, a ausência de interação

Tabela 8: Estimativas das funções locação e dispersão pela análise RFM para o experimento do novo cabo de embreagem.

\begin{tabular}{|c|c|c|c|c|}
\hline CONFIGURAÇÄ́O & RUíD & $\hat{\beta}_{0}$ & $\hat{\beta}_{1}$ & $\hat{\sigma}^{2}$ \\
\hline 1 & 1 & $-1,5$ & 0,820 & 0,15 \\
\hline 2 & -1 & $-1,5$ & 0,907 & 0,10 \\
\hline & 1 & $-1,5$ & 0,820 & 0,15 \\
\hline 3 & -1 & 0,0 & 0,913 & 0,15 \\
\hline & 1 & $-2,0$ & 0,780 & 0,15 \\
\hline 4 & -1 & 0,0 & 0,867 & 0,00 \\
\hline \hline & 1 & $-1,0$ & 0,913 & 0,15 \\
\hline 5 & -1 & $-1,5$ & 0,967 & 0,75 \\
\hline 6 & 1 & $-1,5$ & 0,807 & 0,35 \\
\hline & -1 & $-1,5$ & 0,953 & 0,15 \\
\hline 7 & 1 & $-0,5$ & 0,887 & 0,15 \\
\hline & -1 & $-0,5$ & 0,933 & 0,50 \\
\hline 8 & 1 & $-0,5$ & 0,840 & 0,10 \\
\hline & -1 & $-0,5$ & 0,907 & 0,10 \\
\hline & 1 & $-0,5$ & 0,907 & 0,10 \\
\hline & -1 & $-1,5$ & 0,953 & 0,15 \\
\hline
\end{tabular}


Tabela 9: Estimativas dos coeficientes dos efeitos sobre as funções intercepto, inclinação e variância pela análise RFM do experimento do novo cabo de embreagem

\begin{tabular}{|l|c|c|c|}
\hline \multicolumn{1}{|c|}{ EFEITo } & $\hat{\beta}_{0}$ & $\hat{\beta}_{1}$ & $\hat{\sigma}^{2}$ \\
\hline Constante & $-1,000$ & 0,886 & 0,200 \\
\hline A - Fornecedor da Boccola & $-0,125$ & $-0,013$ & 0,000 \\
\hline B - Fornecedor do Tasselo & 0,063 & $-0,003$ & $-0,075$ \\
\hline C - Terminal & 0,000 & $-0,004$ & $-0,038$ \\
\hline D - Layout & 0,062 & 0,011 & 0,050 \\
\hline E - Tubetto & $-0,063$ & $-0,006$ & $-0,013$ \\
\hline F - Material do Tubetto & $-0,125$ & $-0,026$ & $-0,062$ \\
\hline G - Massa Smorzante & $-0,313$ & $-0,018$ & 0,038 \\
\hline N - Ruído & $-0,125$ & $-0,039$ & $-0,038$ \\
\hline AN - Fornecedor da Boccola*Ruído & $-0,250$ & $-0,001$ & $-0,013$ \\
\hline BN - Fornecedor do Tasselo*Ruído & 0,063 & 0,003 & 0,038 \\
\hline CN - Terminal*Ruído & 0,000 & 0,006 & 0,012 \\
\hline DN - Layout*Ruído & 0,188 & 0,007 & $-0,075$ \\
\hline EN - Tubetto*Ruído & $-0,062$ & $-0,007$ & 0,025 \\
\hline FN - Material do Tubetto*Ruído & $-0,125$ & $-0,009$ & 0,088 \\
\hline GN - Massa Smorzante*Ruído & 0,312 & $-0,003$ & $-0,012$ \\
\hline
\end{tabular}

Figura 5: Gráfico de probabilidade normal para os efeitos de $\hat{\beta}_{0}$ pela abordagem RFM para o experimento do novo cabo de embreagem

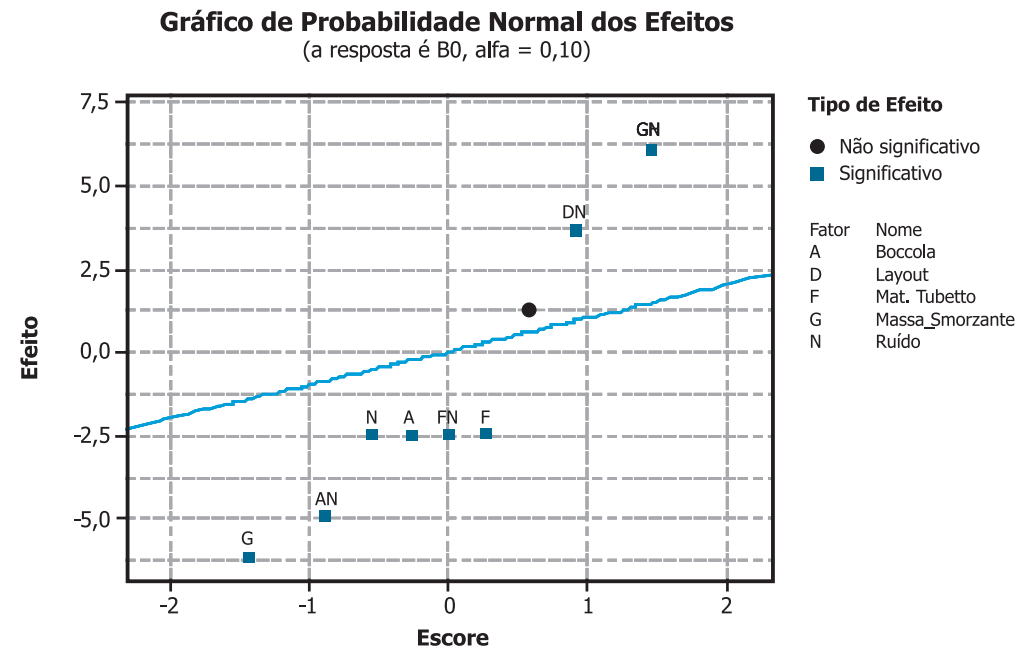


entre os fatores de controle foi assumida. Essa suposição foi feita visto que estudos anteriores e o conhecimento técnico das equipes de projeto e processo em relação a sistemas similares indicaram que essas interações, se existentes, seriam desprezíveis. Essa suposição mostrou-se verdadeira, uma vez que nos experimentos confirmatórios com cabos montados segundo a configuração ótima selecionada observou-se um ganho extraordinário de rendimento do sistema

Figura 6: Gráfico de probabilidade normal para os efeitos de $\hat{\beta}_{1}$ pela abordagem RFM para o experimento do novo cabo de embreagem

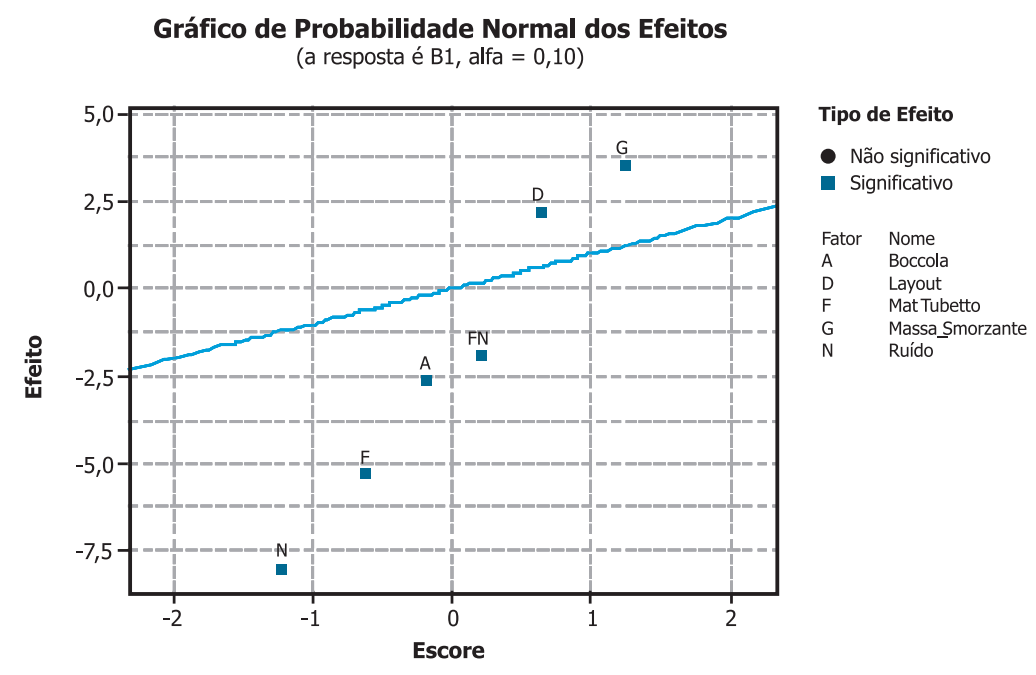

Figura 7: Gráfico de probabilidade normal para os efeitos de $\hat{\sigma}^{2}$ pela abordagem RFM para o experimento do novo cabo de embreagem

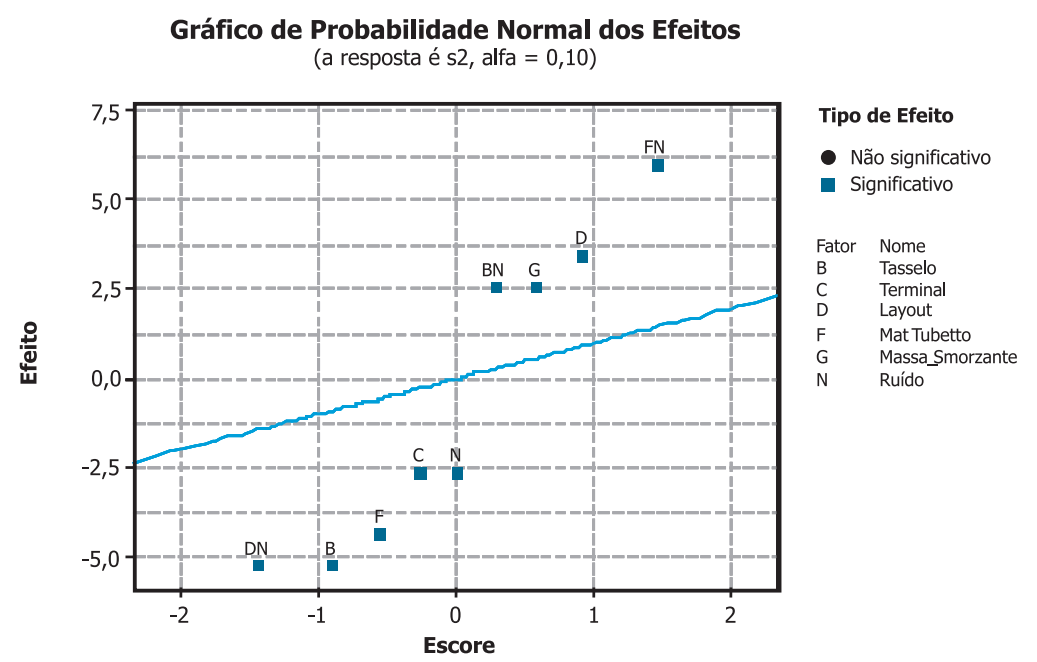


e redução drástica da variabilidade funcional do mesmo (BORGES; FREITAS, 2005).

\section{CONCLUSÃO E CONSIDERAC̣̃̃ES FINAIS}

A situação estudada é um sistema do tipo sinal-resposta e com múltiplos alvos, cuja relação ideal entre o sinal e a resposta é linear simples. Para o caso específico de um sistema de medição visando o levantamento de uma curva de calibração, Miller e Wu (1991) consideraram a utilização de um modelo de efeitos aleatórios. Neste caso, tanto a inclinação quanto a variância do erro foram consideradas aleatórias e modeladas em função dos fatores de controle e ruído. Miller (2002) apresentou, também, para o caso onde a relação entre sinal e resposta era uma reta, um método de análise gráfica dos resultados (joint effects plot).

Entretanto, em muitas situações práticas a relação pode ser mais complexa. As abordagens apresentadas neste trabalho podem ser aplicadas nestas situações, com as devidas adaptações. Miller e Wu (1996) consideraram uma situação prática em que a relação sinal-resposta tinha componentes quadráticos. A análise foi feita através das abordagens PMM e RFM. A segunda mostrou-se mais informativa. Ainda nesta linha, Nair, Taam e Ye (2002) analisaram dados referentes a um processo de moldagem por injeção utilizando regressão funcional.

Neste artigo, estudaram-se e compararam-se os resulta- dos de três propostas de modelagem e análise de dados a uma situação na qual a relação sinal-resposta é linear simples. $\mathrm{Na}$ análise da aplicação prática do novo cabo de embreagem, os efeitos significativos e as combinações ótimas identificadas foram os mesmos para as funções intercepto e inclinação nas três metodologias. As conclusões tornaram-se diferentes quando se analisou a variância do processo. Os métodos RFM e RM forneceram informação adicional de como a variância do processo pode ser minimizada. O método RFM permitiu avaliar o efeito do fator de ruído sobre as funções intercepto, inclinação e variância, buscando por uma combinação ótima, de forma que o ruído afetasse o menos possível nas funções, ou pelo menos, avaliar o quanto que o ruído influenciava os valores dessas funções. $\mathrm{O}$ método RM permitiu dividir a variância total do processo em duas, e verificar que a variância se deve aos níveis dos fatores de ruído, e não ao erro-puro. Porém, o modelo ajustado para a variância no método RM foi mais confuso com relação aos modelos dos demais métodos.

Em um artigo mais recente, Bae e Tsui (2006) apresentam uma generalização do método Modelagem da Resposta (RM) baseando em modelos lineares generalizados. Segundo os autores, tal método proposto fornece resultados mais confiáveis através da modelagem iterativa dos resíduos do modelo ajustado para a resposta. Sugere-se, para um trabalho futuro, o estudo desse método e a comparação de seus resultados com os dos três métodos estudados neste trabalho.

\section{Artigo recebido em 27/06/2007 Aprovado para publicação em 22/11/2007}

\section{- Referências}

BAE, S. J.; TSUI, K. L. Analysis of Dynamic Robust Design Experiment with Explicit \& Hidden Noise Variables. Quality Technology \& Quantitative Management, 1, p. 55-75, 2006.

BORGES, M. F; FREITAS, M. A. Engenharia Robusta de Parâmetros na Indústria Automobilística buscando projetar certo da primeira vez. Anais do V CBGDP Curitiba, PR, Brasil, 2005 (em CD).

BOX, G. Signal-to-noise ratios, performance criteria, and transformations. Technometrics, 30, p. 1-40, 1988.

BOX, G. E. P.; JONES, S. Designs for Minimizing the Effects of Environmental Variables. Technical Report, University of Wisconsin, 1990.
DeMATES, J. (1990). Dynamic analysis of injection molding using Taguchi methods. In Eighth Symposium on Taguchi Methods, p. 313-331. American Supplier Institute, Dearborn, MI.

ENGEL, J.; HUELE, A. F. A generalized linear modeling approach to robust design. Technometrics, 38, p. 365-373, 1996.

FOWLKES, W. Y.; CREVELING, C. M. Engineering Methods for Robust Product Design. Reading, MA: Addison-Wesley, 1995.

GREGO, J. M. Generalized linear models and process variation. Journal of Quality Technology, 25, p. 288-295, 1993.
GROVE, D. M.; DAVIS, T. P. Engineering Quality \& Experimental Design. Essex: Longman Scientific \& Technical, 1992.

HAMADA, M. S; WU, C. F. J. Analysis of Designed Experiments with Complex Aliasing. Journal of Quality Technology, 24 p. 130-137, 1992.

KACKER, R. N. Off-Line Quality Control, Parameter Design, and the Taguch Method. Journal of Quality Technology, 17, p. $176-209,1985$.

LEE, Y.; NELDER, J. A. Robust design via generalized linear models. Journal of Quality Technology, 35, p. 2-12, 2003.

LENTH, R. V. Quick and easy analysis of unreplicated experiments. Technometrics 31, p. 469-473, 1989.
McCASKEY, S. D.; TSUI, K. L. Analysis of dynamic robust design experiments. International Journal of Production Research, 35, p. 1561-1574, 1997.

MANDEL, J. The Statistical Analysis of Experimental Data. New York: Interscience, 1964

MILLER, A.; WU, C. F. J. Improving a Calibration System Through Designed Experiments. Technical Report, University of Waterloo, IIQP, 1991.

MILLER, A.; WU, C. F. J. Parameter Design for Signal-Response Systems: A Different Look at Taguchi's Dynamic Parameter Design. Statistical Science, 11, p. 122136, 1996. 


\section{- Referências}

MILLER, A. Analysis of Parameter Design Experiments for Signal-Response Systems. Journal of Quality Technology, 34, p. 139-151, 2002.

NAIR, V. N. e SHOEMAKER, A. C. The Role of Experimentation in Quality Engineering: A Review of Taguchi's Contributions. Statistical Design and Analysis of Industrial Experiments, ed. S. Ghosh, New York: Marcel Dekker, p. 247-277, 1990

NAIR, V. N. (Editor). Taguchi's parameter design: a panel discussion. Technomerics, 34, 127-161, 1992.
NAIR, V.; TAAM, W.; YE, Q. Analysis of Functional Responses from Robust Design Experiments. Journal of Quality Technology, 34, p. 355-370, 2002.

PHADKE, M. S. Quality Engineering Using Robust Design. Englewood Cliffs, NJ: Prentice-Hall, 1989.

ROSS, P. J. Taguchi Techniques for Quality Engineering. New York, NY: McGrawHill, 1988.

SHOEMAKER, A. C.; TSUI, K. L; WU, C. F. J. Economical experimentation methods for robust design. Technometrics, 33, p. 415-427, 1991.
SHOEMAKER, A. C.; TSUI, K. L; WU, C. F. J. Response model analysis for robust design. Communications in Statistics, 22, p. 1037-1064, 1993.

STEINBERG, D. M.; BURSZTYN, D. Dispersion effects in robust design with noise factors. Journal of Quality Technology, 26, p. 12-20, 1994.

TAGUCHI, G. Introduction to Quality Engineering: Designing Quality into Products and Process. Tokyo, Japan: Asian Productivity Organization, 1986.

TAGUCHI, G.; CHOWDHURY, S.; TAGUCHI, S. Robust Engineering. New York, NY: McGraw Hill, 2000.
TSUI, K. L. Avoiding unnecessary bias in robust design analysis. Computational Statistics and Data Analysis, p. 535-546, 1994.

TSUI, K. L. Response model analysis of dynamic robust design experiments. IIE Transactions, 31, p. 115-131, 1999.

WILKINS, J. O. Putting Taguchi Methods to Work to Solve Design Flaws. Quality Progress, 33, p. 55-59, 2000.

WU, D. F. J.; HAMADA, M. S. Experiments: Planning, Analysis, and Parameter Design Optimization. New York, NY: John Wiley and Sons, 2000.

\section{- Agradecimentos}

Deseja-se reconhecer o papel dos referees anônimos pelo seu meticuloso trabalho, que contribuiu de modo expressivo para a forma final deste artigo.

\section{- Sobre os autores}

\section{Marta Afonso Freitas, Dra.}

Departamento de Engenharia de Produção (UFMG)

Universidade Federal de Minas Gerais

Escola de Engenharia. Departamento de Engenharia de Produção

End.: Av. Antonio Carlos 6627, Campus da Pampulha - Caixa Postal 209 - CEP 30161-970 - Belo Horizonte - MG

Tel: (031) 3409-4899

Email: marta@dep.ufmg.br

\section{Rosiane Mary Rezende Faleiro}

Mestre em Estatística (UFMG)

Email: rosianefaleiro@yahoo.com.br

\section{Marco Fábio Borges}

Mestre em Eng.de Produção (UFMG)

Email: mborges9@ford.com 